\title{
Medical education research and the ethics of different publication models
}

\author{
Muhamad Saiful Bahri Yusoff $^{1}$, Lynn Monrouxe ${ }^{2}$ \\ ${ }^{1}$ Department of Medical Education, School of Medical Sciences, Universiti Sains Malaysia, 16150 Kota Bharu, \\ Kelantan, Malaysia. ${ }^{2}$ Institute of Medical Education, Cardiff University School of Medicine, Cochrane Medical \\ Education Centre, Heath Park, Cardiff, United Kingdom.
}

\begin{tabular}{ll}
\hline ARTICLE INFO \\
Received & $: 20 / 05 / 2014$ \\
Accepted & $: 27 / 06 / 2014$ \\
Published & $: 01 / 09 / 2014$
\end{tabular}

\section{KEYWORD}

Medical education research Publication ethics

Publication models

\section{ABSTRACT}

Journal business models are basically classified based on the source of income to cover publication costs and in general there are two main journal business models which are the toll-access and open-access. These leading to a question that still remains around the ethics of publishing academic work across the different journal business models in terms of (a) editorial decision-making and (b) the dissemination of research that has not been appropriately peer-reviewed for quality and rigor. This paper discussed about these two areas based on the literature and the authors' observations.

(C) Medical Education Department, School of Medical Sciences, Universiti Sains Malaysia. All rights reserved.

CORRESPONDING AUTHOR: Dr. Muhamad Saiful Bahri Yusoff, Department of Medical Education, School of Medical Sciences, Universiti Sains Malaysia, 16150 Kota Bharu, Kelantan, Malaysia.

E-mail: msaiful_bahri@usm.my

\section{Introduction}

In 2009, the freelance researcher Phil Davis had a paper accepted by 'The Open-Information Science Journal' after being 'in review' for four months (1). In August 2012, a 'Professor Marcie Rathke' at the 'University of Southern North Dakota at Hoople' had a manuscript accepted by Advances in Pure Mathematics after only 10 days in review (2). What do these two events have in common? The answer is that they are both 'spoof' papers created by the computer programmes (SCIjen and Mathgen respectively): both manuscripts were grammatically correct, but completely nonsensical and both 'authors' were asked to pay publishing costs (USD\$500800) (1, 2). While neither article was published as the 'authors' subsequently retracted them, they do raise a number of serious questions for researchers in our current 'publish or perish' culture of academia (3). Although these particular cases cannot be generalized to all open access journals, a question still remains around the ethics of publishing academic work across the different journal business models in terms of (a) editorial decision-making and (b) the dissemination of research that has not been appropriately peer-reviewed for quality and rigor.

\section{Publishing models}

In a nutshell, journal business models are basically classified based on the source of income to cover publication costs. In general there are two main journal business models: the toll-access and open-access (4). The toll-access model recovers cost of publication by subscription fee to the content of the journal and it is the most common model adopted by publishers $(4,5)$. Gaining popularity is the openaccess model. This model recovers cost of 
publication through an article processing fee in advance of publication which is paid either by individual author, the author's funding agency, the author's employing institution or sponsors of the journal $(4,5)$. The open-access model allows the content of the journal to be available to all users without any fee.

In addition to these two main models there is the hybrid open-access system (5). This model makes articles freely available to users conditionally. For example, toll-access publishers sometimes provide free access to articles for a period of time (say, after one year), sometimes toll-access publishers provide a choice to authors (post acceptance) to make their articles freely accessible by paying some amount of fee, and sometimes toll-access publishers provide free access to old journal volumes or pre-prints of articles (5). At present, there are a growing number of publishers who are adopting this hybrid model.

The Committee on Publication Ethics (COPE) is an advisory body that informs editors and publishers of peer reviewed journals about aspects of good practice, irrespective of business model behind the published journal. Amongst other things, COPE emphasizes that journal editors should strive to meet authors' and readers' needs, have the processes in hand to assure the quality of published materials, and must not compromise intellectual and ethical standards (6). It is to these ethical standards that we now turn.

\section{Ethics of open-access: the case for}

While citations across the different models are comparable (7-10), several articles have shown that an open-access model is at advantage in terms of accessing a wider readership than the toll-access model, especially in the developing countries (11). The toll-based model of publication tends to limit access to institutions or individuals who have the resources to pay for subscription costs. Thus a key benefit to publishing in an open access forum is that it potentially reaches a wider audience, including a non-research active one (e.g. students, lecturers, curriculum developers). Indeed, making our research available to such a wider audience is a key of the requirements of ethical research: it is a morally correct approach that enables funders (i.e. the general public) to both access and use the research that they have paid for. Such public involvement is of crucial importance to researchers in low-income countries who have an obligation to share the outcome of their work with their colleagues thus increasing the 'social value'. Social value includes aspects such as reaching the beneficiaries of the research, the mechanisms to engage research with society, and ensuring that the conduct of research did not undermine the community's existing values (12). Another argument in favour of open-access publishing is that it has been argued that tollmodels are unjust. They exploit the labour of publically-funded academics who (a) submit their (publically-funded) research manuscripts to a journal run by a commercial enterprise; (b) spend publically-funded time carrying out the peer-review of these manuscripts; (c) work as (often-unpaid) members of editorial boards; and (d) the publically-funded institutions then pay for access to the "fruits of their own intellectual labour". Thus within the toll-model of publishing, the publishers profit unfairly from such 'free-labour' (13).

Finally, the issue of creativity has been discussed in the toll- vs. open-access debate. Here, tollmodels have been branded as being relatively conservative though their rigidity in areas such as disciplinary boundaries and publication formats. Such practices constrict intellectual and scholarly thinking and thus inhibiting creativity. Open-access models, on the other hand, can offer the possibility of moving away from such an overly rigid approach with 'open access initiatives have the potential to lead to the development of new, creative and more productive communities of publishing practice and the generation of new forms of understanding and research collaboration' (13, 14). 


\section{Ethics of open-access: the case against}

There has been a suggestion that the open-access model of publishing should facilitate more effective and better quality research. But, does this claim hold true? Regardless of the journal business model, the majority of editors heavily rely on peer-reviewers' comment to inform their judgments about the quality of the manuscripts submitted. However, the decision-making process is not straightforward. Such decisionmaking can become complex due to the fact that human judgment is affected by multiple factors (15). Some of factors are under control: for example, the editorial process, the scope of the journal, the review process, the review forms and triangulation in decision-making. Conversely, some of factors are out of one's control. For example, the available funding to cover publication cost, the impact of monetary incentives on the editor's judgment, the psychological pressure to meet publishing demands, the generosity bias (i.e. tendency to provide more positive comment), and changes in publishing policy. As mentioned previously, journals require substantial amount of income to cover their publication cost. The toll-access journals heavily rely on their quality of papers to attract subscription to their journal so that they could gain a sustainable income (16). Therefore, rationally, editors are under pressure to publish papers with the highest quality: this factor contributes to upholding strong intellectual and ethical standards. On the other hand, income for the open-access journals completely depends on the one-off fee in advance of publication: the number of subscriptions they attract will not increase their income (5). Mathematically, openaccess journals need to increase the number of papers they publish in order to increase their income. Thus unlike toll-access journals, the quality of the papers they publish tend not to affect the actual income they receive.

Peer review is a cultural construct, embedded within our academic culture, it provides us with a 'measure' of authenticity and rigour. If you work in an academic institution, it is your responsibility to peer review professionally, intellectually and ethically. As peer-reviewers of both journal models, our personal experiences in acting for both toll- and open-access journals is that the open-access journals can sometimes be more 'merciful' towards publishing 'borderline' papers than toll-access journals. For example, we have experienced situations where editors have pressurized us to accept manuscripts, despite us saying 'reject' more than once (by overturning rejections and requesting multiple revisions, and once accepting a paper that had been firmly rejected because of serious concerns over the rigor of the work). Therefore, as peer-reviewers we can sometimes be inadvertently implicated in these ethical issues: the publication of dubious research may lead to 'a chain reaction' of compromised scientific merits by circulating lesser-quality papers to end-users, which later may implicate on the best and evidence-based practices.

\section{Concluding remarks: the way forward for medical education}

At present, there are a growing number of medical education researchers trying to publish within a relatively small number of toll-model journals, meaning a high rejection rate for authors, with some good quality research potentially being unpublished alongside work that is 'unpublishable'. For instances, it was estimated that more than 1.2 millions of articles (from various fields) received by a publisher per year, but very few of them were accepted for publication. This situation has created a market for open-access journals to be developed within medical education to capitalise on this. Furthermore, in this 'publish or perish' culture, while most authors prefer to submit their manuscripts to journals they perceived as being high quality $(16,17)$, some researchers purposively target open access journals knowing that their papers are of lesser quality (16). With this rise in open-access medical education journals, will this result in the potential demise of high-quality medical education research? For that reason, we advocate more research should be conducted in future to investigate potential impacts of different journal business models on publication ethics as a part of quality indicators of medical education journals. Within limited 
data, it seems that the toll-accessed journals were perceived as more reliable model than the openaccess journals to uphold intellectual and ethical standards.

Last but not least, in line with the COPE and DORA statements to assure the quality of published materials $(6,18)$, Randy Schekman - a nobel prize-winning Biologist - echoed, "Big journals' reputations are only partly warranted. While they publish many outstanding papers, they do not publish only outstanding papers. Neither are they the only publishers of outstanding research. It is the quality of the science, not the journal's brand, that matters (14)."

\section{Reference}

1. Davis P. Open access publisher accepts nonsense manuscript for dollars. The Scholarly Kitchen; 2009 [cited 201328 November]; Available from: http://scholarlykitchen.sspnet.org/2009/06/1 0/nonsense-for-dollars/.

2. Rathke M. Mathgen paper accepted! 2012; Available from: http://thatsmathematics.com/blog/archives/1 02.

3. Parchomovsky G. Publish or perish. Michigan Law Review. 2000;98(4):926-52.

4. Knowledge Exchange. Knowledge exchange briefing paper on journal business models2009: Available from: http://www.knowledgeexchange.info/Files/Filer/downloads/Open\% 20Access/KE_Briefing_Paper_on_Journal_ Business_Models.pdf.

5. Bernius S, Hanauske M, Konig W, Dugall B. Open access models and their implications for the players on the scientific publishing market. Economic Analysis and Policy. 2009;39(1):103-15.

6. COPE. Code of conduct and best practice guidelines for journal editors2013.

7. Bjork B. A comparison of subscription and open access journals in construction management and related fields Australasian Journal of Construction Economics and Building. 2012;12(2):27-42.
8. Björk B-C, Solomon D. Open access versus subscription journals: a comparison of scientific impact. Bmc Medicine. 2012;10(1):73-82.

9. Davis PM. Open access, readership, citations: a randomized controlled trial of scientific journal publishing. The FASEB Journal. 2011;25(7):2129-34.

10. Davis PM, Walters WH. The impact of free access to the scientific literature: a review of recent research. Journal of the Medical Library Association: JMLA. 2011;99(3):208-17.

11. Evans JA, Reimer J. Open access and global participation in science. Science. 2009;323(5917):1025.

12. Emanuel EJ, Wendler D, Killen J, Grady C. What makes clinical research in developing countries ethical? The benchmarks of ethical research. Journal of Infectious Diseases. 2004;189(5):930-7.

13. Parker M. The ethics of open access publishing. BMC medical ethics. 2013;14(1):16.

14. Schekman R. How journals like Nature, Cell and Science are damaging science. The Guardian. 2013.

15. Albanese MA. Challenges in using rater judgements in medical education. Journal of evaluation in clinical practice. 2000;6(3):305-19.

16. Schroter S, Tite L, Smith R. Perceptions of open access publishing: interviews with journal authors. BMJ: British Medical Journal. 2005;330(7494):756.

17. Schroter S, Tite L. Open access publishing and author-pays business models: a survey of authors' knowledge and perceptions. JRSM. 2006;99(3):141-8.

18. DORA. Declaration on Research Assessment. San Franscisco: ASCB Annual Meeting; 2012 [25th November 2013]. 